\title{
Weighted Ostrowski type inequalities for co-ordinated convex functions
}

\author{
Hüseyin Budak ${ }^{1 *}$ (ID
}

\section{"Correspondence:}

hsyn.budak@gmail.com

${ }^{1}$ Department of Mathematics,

Faculty of Science and Arts, Düzce

University, Düzce, Turkey

\section{Springer}

\begin{abstract}
In this paper, utilizing an identity given by Yıldız and Sarıkaya in (Yildiz and Sarikaya in Int. J. Anal. Appl. 13(1):64-69, 2017), we establish some weighted Ostrowski type inequalities for co-ordinated convex functions in a rectangle from the plane $\mathbb{R}^{2}$. Moreover, as special cases of our main results, we give some weighted Hermite-Hadamard type inequalities. The results given in this paper provide generalizations of some result established in earlier works.
\end{abstract}

MSC: 26D07; 26D10;26D15; 26B15; 26B25

Keywords: Ostrowski inequality; Hermite-Hadamard-Fejer inequality; Co-ordinated convex; Integral inequalities

\section{Introduction}

In the history of calculus development, integral inequalities have been thought of as a key factor in the theory of differential and integral equations. The study of various types of integral inequalities has been in the focus of great attention of a number of scientists interested in both pure and applied mathematics for more than a century. One of the many fundamental mathematical discoveries of A. M. Ostrowski [15] is the following classical integral inequality associated with the differentiable mappings:

Let $\mathcal{F}:\left[\rho_{1}, \rho_{2}\right] \rightarrow \mathbb{R}$ be a differentiable mapping on $\left(\rho_{1}, \rho_{2}\right)$ whose derivative $\mathcal{F}^{\prime}$ : $\left(\rho_{1}, \rho_{2}\right) \rightarrow \mathbb{R}$ is bounded on $\left(\rho_{1}, \rho_{2}\right)$, i.e., $\left\|\mathcal{F}^{\prime}\right\|_{\infty}=\sup \left|\mathcal{F}^{\prime}(\psi)\right|<\infty$. Then, the inequality holds:

$$
\left|\mathcal{F}(\kappa)-\frac{1}{\rho_{2}-\rho_{1}} \int_{\rho_{1}}^{\rho_{2}} \mathcal{F}(\psi) d \psi\right| \leq\left[\frac{1}{4}+\frac{\left(\kappa-\frac{\rho_{1}+\rho_{2}}{2}\right)^{2}}{\left(\rho_{2}-\rho_{1}\right)^{2}}\right]\left(\rho_{2}-\rho_{1}\right)\left\|\mathcal{F}^{\prime}\right\|_{\infty}
$$

for all $\kappa \in\left[\rho_{1}, \rho_{2}\right]$. The constant $\frac{1}{4}$ is the best possible.

The other important fundamental result, the Hermite-Hadamard inequality discovered by C. Hermite and J. Hadamard (see, e.g., [7], [18, p. 137]), is one of the most wellestablished inequalities in the theory of convex functions with a geometrical interpretation and many applications. These inequalities state that if $\mathcal{F}: I \rightarrow \mathbb{R}$ is a convex function

(c) The Author(s) 2022. This article is licensed under a Creative Commons Attribution 4.0 International License, which permits use, sharing, adaptation, distribution and reproduction in any medium or format, as long as you give appropriate credit to the original author(s) and the source, provide a link to the Creative Commons licence, and indicate if changes were made. The images or other third party material in this article are included in the article's Creative Commons licence, unless indicated otherwise in a credit line to the material. If material is not included in the article's Creative Commons licence and your intended use is not permitted by statutory regulation or exceeds the permitted use, you will need to obtain permission directly from the copyright holder. To view a copy of this licence, visit http://creativecommons.org/licenses/by/4.0/. 
on the interval $I$ of real numbers and $\rho_{1}, \rho_{2} \in I$ with $\rho_{1}<\rho_{2}$, then

$$
\mathcal{F}\left(\frac{\rho_{1}+\rho_{2}}{2}\right) \leq \frac{1}{\rho_{2}-\rho_{1}} \int_{\rho_{1}}^{\rho_{2}} \mathcal{F}(\kappa) d \kappa \leq \frac{\mathcal{F}\left(\rho_{1}\right)+\mathcal{F}\left(\rho_{2}\right)}{2}
$$

Both inequalities hold in the reversed direction if $\mathcal{F}$ is concave. We note that the HermiteHadamard inequality may be regarded as a refinement of the concept of convexity, and it follows easily from Jensen's inequality. The Hermite-Hadamard inequality for convex functions has received renewed attention in recent years, and a remarkable variety of refinements and generalizations have been studied.

A formal definition for co-ordinated convex function may be stated as follows:

Definition 1 A function $\mathcal{F}: \Delta:=\left[\rho_{1}, \rho_{2}\right] \times\left[\rho_{3}, \rho_{4}\right] \rightarrow \mathbb{R}$ is called co-ordinated convex on $\Delta$, for all $(\kappa, u),(\gamma, v) \in \Delta$ and $\psi, \varphi \in[0,1]$, if it satisfies the following inequality:

$$
\begin{aligned}
& \mathcal{F}(\psi \kappa+(1-\psi) \gamma, \varphi u+(1-\varphi) v) \\
& \quad \leq \psi \varphi \mathcal{F}(\kappa, u)+\psi(1-\varphi) \mathcal{F}(\kappa, v)+\varphi(1-\psi) \mathcal{F}(\gamma, u)+(1-\psi)(1-\varphi) \mathcal{F}(\gamma, v) .
\end{aligned}
$$

The mapping $\mathcal{F}$ is a co-ordinated concave on $\Delta$ if inequality (1.1) holds in reversed direction for all $\psi, \varphi \in[0,1]$ and $(\kappa, u),(\gamma, v) \in \Delta$.

Barnet and Dragomir gave the following Ostrowski type inequalities for double integrals in [5].

Theorem 1 Let $\mathcal{F}: \Delta:=\left[\rho_{1}, \rho_{2}\right] \times\left[\rho_{3}, \rho_{4}\right] \rightarrow \mathbb{R}$ be continuous on $\Delta, \mathcal{F}_{\kappa, \gamma}^{\prime \prime}=\frac{\partial^{2} \mathcal{F}}{\partial \kappa \partial \gamma}$ exists on $\left(\rho_{1}, \rho_{2}\right) \times\left(\rho_{3}, \rho_{4}\right)$ and is bounded, i. e.,

$$
\left\|\mathcal{F}_{\kappa, \gamma}^{\prime \prime}\right\|_{\infty}=\sup _{(\kappa, \gamma) \in\left(\rho_{1}, \rho_{2}\right) \times\left(\rho_{3}, \rho_{4}\right)}\left|\frac{\partial^{2} \mathcal{F}(\kappa, \gamma)}{\partial \kappa \partial \gamma}\right|<\infty .
$$

Then, we have the inequality:

$$
\begin{aligned}
& \mid \int_{\rho_{1}}^{\rho_{2}} \int_{\rho_{3}}^{\rho_{4}} \mathcal{F}(\psi, \varphi) d \varphi d \psi-\left(\rho_{2}-\rho_{1}\right)\left(\rho_{4}-\rho_{3}\right) \mathcal{F}(\kappa, \gamma) \\
& \quad-\left[\left(\rho_{2}-\rho_{1}\right) \int_{\rho_{3}}^{\rho_{4}} \mathcal{F}(\kappa, \varphi) d \varphi+\left(\rho_{4}-\rho_{3}\right) \int_{\rho_{1}}^{\rho_{2}} \mathcal{F}(\psi, \gamma) d \psi\right] \mid \\
& \quad \leq\left[\frac{1}{4}\left(\rho_{2}-\rho_{1}\right)^{2}+\left(\kappa-\frac{\rho_{1}+\rho_{2}}{2}\right)^{2}\right]\left[\frac{1}{4}\left(\rho_{4}-\rho_{3}\right)^{2}+\left(\gamma-\frac{\rho_{3}+\rho_{4}}{2}\right)^{2}\right]\left\|\mathcal{F}_{\kappa, \gamma}^{\prime \prime}\right\|_{\infty},
\end{aligned}
$$

for all $(\kappa, \gamma) \in \Delta$.

In [6], Dragomir proved the following inequalities which are the Hermite-Hadamard type inequalities for co-ordinated convex functions on the rectangle from the plane $\mathbb{R}^{2}$.

Theorem 2 Suppose that $\mathcal{F}: \Delta \rightarrow \mathbb{R}$ is co-ordinated convex, then we have the following inequalities:

$$
\mathcal{F}\left(\frac{\rho_{1}+\rho_{2}}{2}, \frac{\rho_{3}+\rho_{4}}{2}\right)
$$




$$
\begin{aligned}
\leq & \frac{1}{2}\left[\frac{1}{\rho_{2}-\rho_{1}} \int_{\rho_{1}}^{\rho_{2}} \mathcal{F}\left(\kappa, \frac{\rho_{3}+\rho_{4}}{2}\right) d \kappa+\frac{1}{\rho_{4}-\rho_{3}} \int_{\rho_{3}}^{\rho_{4}} \mathcal{F}\left(\frac{\rho_{1}+\rho_{2}}{2}, \gamma\right) d \gamma\right] \\
\leq & \frac{1}{\left(\rho_{2}-\rho_{1}\right)\left(\rho_{4}-\rho_{3}\right)} \int_{\rho_{1}}^{\rho_{2}} \int_{\rho_{3}}^{\rho_{4}} \mathcal{F}(\kappa, \gamma) d \gamma d \kappa \\
\leq & \frac{1}{4}\left[\frac{1}{\rho_{2}-\rho_{1}} \int_{\rho_{1}}^{\rho_{2}} \mathcal{F}\left(\kappa, \rho_{3}\right) d \kappa+\frac{1}{\rho_{2}-\rho_{1}} \int_{\rho_{1}}^{\rho_{2}} \mathcal{F}\left(\kappa, \rho_{4}\right) d \kappa\right. \\
& \left.+\frac{1}{\rho_{4}-\rho_{3}} \int_{\rho_{3}}^{\rho_{4}} \mathcal{F}\left(\rho_{1}, \gamma\right) d \gamma+\frac{1}{\rho_{4}-\rho_{3}} \int_{\rho_{3}}^{\rho_{4}} \mathcal{F}\left(\rho_{2}, \gamma\right) d \gamma\right] \\
\leq & \frac{\mathcal{F}\left(\rho_{1}, \rho_{3}\right)+\mathcal{F}\left(\rho_{1}, \rho_{4}\right)+\mathcal{F}\left(\rho_{2}, \rho_{3}\right)+\mathcal{F}\left(\rho_{2}, \rho_{4}\right)}{4} .
\end{aligned}
$$

The above inequalities are sharp. The inequalities in (1.3) hold in the reverse direction if the mapping $\mathcal{F}$ is a co-ordinated concave mapping.

Over the years, many papers have been dedicated to the generalizations and new versions of the inequalities (1.2) and (1.3) using the different types of convex functions. For the other Ostrowski and Hermite-Hadamard type inequalities for co-ordinated convex functions, please refer to ([1-4, 8-14, 16, 17, 19-29])

In [30], Yıldız and Sarıkaya proved the following Lemma.

Lemma 1 Let $w: \Delta:=\left[\rho_{1}, \rho_{2}\right] \times\left[\rho_{3}, \rho_{4}\right] \rightarrow[0, \infty)$ be an integrable function on $\Delta$ and let $\mathcal{F}: \Delta \rightarrow \mathbb{R}$ be an absolutely continuous function such that the partial derivatives of order $\frac{\partial^{2} \mathcal{F}(\psi, \varphi)}{\partial \psi \partial \varphi}$ exist for all $(\psi, \varphi) \in \Delta$. Then we have the identity

$$
\begin{aligned}
& \left(\int_{\rho_{1}}^{\rho_{2}} \int_{\rho_{3}}^{\rho_{4}} w(u, v) d v d u\right) \mathcal{F}(\kappa, \gamma)-\int_{\rho_{1}}^{\rho_{2}} \int_{\rho_{3}}^{\rho_{4}} w(u, v) \mathcal{F}(u, \gamma) d v d u \\
& \quad-\int_{\rho_{1}}^{\rho_{2}} \int_{\rho_{3}}^{\rho_{4}} w(u, v) \mathcal{F}(\kappa, v) d v d u \\
& \quad+\int_{\rho_{1}}^{\rho_{2}} \int_{\rho_{3}}^{\rho_{4}} w(u, v) \mathcal{F}(u, v) d v d u \\
& =\int_{\rho_{1}}^{\rho_{2}} \int_{\rho_{3}}^{\rho_{4}} P(\kappa, \tau ; \gamma, \eta) \frac{\partial^{2} \mathcal{F}(\tau, \eta)}{\partial \psi \partial \varphi} d \eta d \tau
\end{aligned}
$$

where

$$
P(\kappa, \tau ; \gamma, \eta)= \begin{cases}\int_{\rho_{1}}^{\tau} \int_{\rho_{3}}^{\eta} w(u, v) d v d u, & \rho_{1} \leq \tau<\kappa, \rho_{3} \leq \eta<\gamma, \\ \int_{\rho_{1}}^{\tau} \int_{\rho_{4}}^{\eta} w(u, v) d v d u, & \rho_{1} \leq \tau<\kappa, \gamma \leq \eta \leq \rho_{4}, \\ \int_{\rho_{2}}^{\tau} \int_{\rho_{3}}^{\eta} w(u, v) d v d u, & \kappa \leq \tau \leq \rho_{2}, \rho_{3} \leq \eta<\gamma, \\ \int_{\rho_{2}}^{\tau} \int_{\rho_{4}}^{\eta} w(u, v) d v d u, & \kappa \leq \tau \leq \rho_{2}, \gamma \leq \eta \leq \rho_{4} .\end{cases}
$$

The aim of this paper is to establish some weighted generalizations of the Ostrowski type integral inequalities. The results presented in this paper provide extensions of those given in [14].

\section{Weighted Ostrowski type inequalities}

In this section, using Lemma 1, we established some weighted Ostrowski type inequalities for co-ordinated convex mapping. 
First, we define the following mapping

$$
\begin{aligned}
\Theta\left(\rho_{1}, \rho_{2}, \rho_{3}, \rho_{4} ; \mathcal{F}, w\right) \\
=\mathcal{F}(\kappa, \gamma)-\frac{1}{m\left(\rho_{1}, \rho_{2} ; \rho_{3}, \rho_{4}\right)} \int_{\rho_{1}}^{\rho_{2}} \int_{\rho_{3}}^{\rho_{4}} w(u, v) \mathcal{F}(u, \gamma) d v d u \\
\quad-\frac{1}{m\left(\rho_{1}, \rho_{2} ; \rho_{3}, \rho_{4}\right)} \int_{\rho_{1}}^{\rho_{2}} \int_{\rho_{3}}^{\rho_{4}} w(u, v) \mathcal{F}(\kappa, v) d v d u \\
\quad+\frac{1}{m\left(\rho_{1}, \rho_{2} ; \rho_{3}, \rho_{4}\right)} \int_{\rho_{1}}^{\rho_{2}} \int_{\rho_{3}}^{\rho_{4}} w(u, v) \mathcal{F}(u, v) d v d u
\end{aligned}
$$

where

$$
m\left(\rho_{1}, \rho_{2} ; \rho_{3}, \rho_{4}\right)=\int_{\rho_{1}}^{\rho_{2}} \int_{\rho_{3}}^{\rho_{4}} w(u, v) d v d u
$$

Using the change of variables in Lemma 1, we have the following identity:

$$
\begin{aligned}
\Theta\left(\rho_{1}, \rho_{2}, \rho_{3}, \rho_{4} ; \mathcal{F}, p\right) & \frac{\left(\kappa-\rho_{1}\right)\left(\gamma-\rho_{3}\right)}{m\left(\rho_{1}, \rho_{2} ; \rho_{3}, \rho_{4}\right)} \int_{0}^{1} \int_{0}^{1}\left[\int_{\rho_{1}}^{U_{1}(\psi)} \int_{\rho_{3}}^{V_{1}(\varphi)} w(u, v) d v d u\right] \\
& \times \frac{\partial^{2} \mathcal{F}}{\partial \psi \partial \varphi}\left(U_{1}(\psi), V_{1}(\varphi)\right) d \varphi d \psi \\
& +\frac{\left(\kappa-\rho_{1}\right)\left(\rho_{4}-\gamma\right)}{m\left(\rho_{1}, \rho_{2} ; \rho_{3}, \rho_{4}\right)} \int_{0}^{1} \int_{0}^{1}\left[\int_{\rho_{1}}^{U_{1}(\psi)} \int_{\rho_{4}}^{V_{2}(\varphi)} w(u, v) d v d u\right] \\
& \times \frac{\partial^{2} \mathcal{F}}{\partial \psi \partial \varphi}\left(U_{1}(\psi), V_{2}(\varphi)\right) d \varphi d \psi \\
& +\frac{\left(\rho_{2}-\kappa\right)\left(\gamma-\rho_{3}\right)}{m\left(\rho_{1}, \rho_{2} ; \rho_{3}, \rho_{4}\right)} \int_{0}^{1} \int_{0}^{1}\left[\int_{\rho_{2}}^{U_{2}(\psi)} \int_{\rho_{3}}^{V_{1}(\varphi)} w(u, v) d v d u\right] \\
& \times \frac{\partial^{2} \mathcal{F}}{\partial \psi \partial \varphi}\left(U_{2}(\psi), V_{1}(\varphi)\right) d \varphi d \psi \\
& +\frac{\left(\rho_{2}-\kappa\right)\left(\rho_{4}-\gamma\right)}{m\left(\rho_{1}, \rho_{2} ; \rho_{3}, \rho_{4}\right)} \int_{0}^{1} \int_{0}^{1}\left[\int_{\rho_{2}}^{U_{2}(\psi)} \int_{\rho_{4}}^{V_{2}(\varphi)} w(u, v) d v d u\right] \\
& \times \frac{\partial^{2} \mathcal{F}}{\partial \psi \partial \varphi}\left(U_{2}(\psi), V_{2}(\varphi)\right) d \varphi d \psi,
\end{aligned}
$$

where $U_{1}(\psi)=\psi \kappa+(1-\psi) \rho_{1}, U_{2}(\psi)=\psi \kappa+(1-\psi) \rho_{2}, V_{1}(\varphi)=\varphi \gamma+(1-\varphi) \rho_{3}$ and $V_{2}(\varphi)=$ $\varphi \gamma+(1-\varphi) \rho_{4}$.

Theorem 3 Suppose that the mapping $w$ is as in Lemma 1. Moreover, let $w$ is bounded on $\Delta$, i.e., $\|w\|_{\infty}:=\sup _{(\kappa, \gamma) \in \Delta}|w(\kappa, \gamma)|$. If $\left|\frac{\partial^{2} \mathcal{F}}{\partial \psi \partial \varphi}\right|$ is a co-ordinated convex function on $\Delta$, then for all $(\kappa, \gamma) \in \Delta$ we have the following inequality

$$
\begin{aligned}
\mid \Theta & \left(\rho_{1}, \rho_{2}, \rho_{3}, \rho_{4} ; \mathcal{F}, p\right) \mid \\
& \leq \frac{\|w\|_{\infty}}{36 \times m\left(\rho_{1}, \rho_{2} ; \rho_{3}, \rho_{4}\right)}
\end{aligned}
$$




$$
\begin{aligned}
& \times\left\{( \kappa - \rho _ { 1 } ) ^ { 2 } ( \gamma - \rho _ { 3 } ) ^ { 2 } \left[4\left|\frac{\partial^{2} \mathcal{F}}{\partial \psi \partial \varphi}(\kappa, \gamma)\right|+2\left|\frac{\partial^{2} \mathcal{F}}{\partial \psi \partial \varphi}\left(\kappa, \rho_{3}\right)\right|\right.\right. \\
& \left.+2\left|\frac{\partial^{2} \mathcal{F}}{\partial \psi \partial \varphi}\left(\rho_{1}, \gamma\right)\right|+\left|\frac{\partial^{2} \mathcal{F}}{\partial \psi \partial \varphi}\left(\rho_{1}, \rho_{3}\right)\right|\right] \\
& +\left(\kappa-\rho_{1}\right)^{2}\left(\rho_{4}-\gamma\right)^{2}\left[4\left|\frac{\partial^{2} \mathcal{F}}{\partial \psi \partial \varphi}(\kappa, \gamma)\right|+2\left|\frac{\partial^{2} \mathcal{F}}{\partial \psi \partial \varphi}\left(\kappa, \rho_{4}\right)\right|\right. \\
& \left.+2\left|\frac{\partial^{2} \mathcal{F}}{\partial \psi \partial \varphi}\left(\rho_{1}, \gamma\right)\right|+\left|\frac{\partial^{2} \mathcal{F}}{\partial \psi \partial \varphi}\left(\rho_{1}, \rho_{4}\right)\right|\right] \\
& +\left(\rho_{2}-\kappa\right)^{2}\left(\gamma-\rho_{3}\right)^{2}\left[4\left|\frac{\partial^{2} \mathcal{F}}{\partial \psi \partial \varphi}(\kappa, \gamma)\right|+2\left|\frac{\partial^{2} \mathcal{F}}{\partial \psi \partial \varphi}\left(\kappa, \rho_{3}\right)\right|\right. \\
& \left.+2\left|\frac{\partial^{2} \mathcal{F}}{\partial \psi \partial \varphi}\left(\rho_{2}, \gamma\right)\right|+\left|\frac{\partial^{2} \mathcal{F}}{\partial \psi \partial \varphi}\left(\rho_{2}, \rho_{3}\right)\right|\right] \\
& +\left(\rho_{2}-\kappa\right)^{2}\left(\rho_{4}-\gamma\right)^{2}\left[4\left|\frac{\partial^{2} \mathcal{F}}{\partial \psi \partial \varphi}(\kappa, \gamma)\right|+2\left|\frac{\partial^{2} \mathcal{F}}{\partial \psi \partial \varphi}\left(\kappa, \rho_{4}\right)\right|\right. \\
& \left.\left.+2\left|\frac{\partial^{2} \mathcal{F}}{\partial \psi \partial \varphi}\left(\rho_{2}, \gamma\right)\right|+\left|\frac{\partial^{2} \mathcal{F}}{\partial \psi \partial \varphi}\left(\rho_{2}, \rho_{4}\right)\right|\right]\right\}
\end{aligned}
$$

where the mapping $\Theta$ is defined as in (2.1).

Proof By taking the modulus of the equality (2.2), we have

$$
\begin{aligned}
\left|\Theta\left(\rho_{1}, \rho_{2}, \rho_{3}, \rho_{4} ; \mathcal{F}, p\right)\right| & \left(\kappa-\rho_{1}\right)\left(\gamma-\rho_{3}\right) \\
= & \frac{m\left(\rho_{1}, \rho_{2} ; \rho_{3}, \rho_{4}\right)}{U_{1}(\psi)} \int_{\rho_{3}}^{V_{1}(\varphi)} w(u, v) d v d u|| \frac{\partial^{2} \mathcal{F}}{\partial \psi \partial \varphi}\left(U_{1}(\psi), V_{1}(\varphi)\right) \mid d \varphi d \psi \\
& \left.\times \int_{0}^{1} \int_{0}^{1} \mid \int_{\rho_{1}}^{U}\right) \\
& +\frac{\left(\kappa-\rho_{1}\right)\left(\rho_{4}-\gamma\right)}{m\left(\rho_{1}, \rho_{2} ; \rho_{3}, \rho_{4}\right)} \\
& \times \int_{0}^{1} \int_{0}^{1}\left|\int_{\rho_{1}}^{U_{1}(\psi)} \int_{\rho_{4}}^{V_{2}(\varphi)} w(u, v) d v d u\right|\left|\frac{\partial^{2} \mathcal{F}}{\partial \psi \partial \varphi}\left(U_{1}(\psi), V_{2}(\varphi)\right)\right| d \varphi d \psi \\
& +\frac{\left(\rho_{2}-\kappa\right)\left(\gamma-\rho_{3}\right)}{m\left(\rho_{1}, \rho_{2} ; \rho_{3}, \rho_{4}\right)} \\
& \times \int_{0}^{1} \int_{0}^{1}\left|\int_{\rho_{2}}^{U_{2}(\psi)} \int_{\rho_{3}}^{V_{1}(\varphi)} w(u, v) d v d u\right|\left|\frac{\partial^{2} \mathcal{F}}{\partial \psi \partial \varphi}\left(U_{2}(\psi), V_{1}(\varphi)\right)\right| d \varphi d \psi \\
& +\frac{\left(\rho_{2}-\kappa\right)\left(\rho_{4}-\gamma\right)}{m\left(\rho_{1}, \rho_{2} ; \rho_{3}, \rho_{4}\right)} \\
& \times \int_{0}^{1} \int_{0}^{1}\left|\int_{\rho_{2}}^{U_{2}(\psi)} \int_{\rho_{4}}^{V_{2}(\varphi)} w(u, v) d v d u\right|\left|\frac{\partial^{2} \mathcal{F}}{\partial \psi \partial \varphi}\left(U_{2}(\psi), V_{2}(\varphi)\right)\right| d \varphi d \psi .
\end{aligned}
$$

Since $w(\kappa, \gamma)$ is bounded on $\Delta$, and $\left|\frac{\partial^{2} \mathcal{F}}{\partial \psi \partial \varphi}\right|$ is co-ordinated convex on $\Delta$, we obtain

$$
\int_{0}^{1} \int_{0}^{1}\left|\int_{\rho_{1}}^{U_{1}(\psi)} \int_{\rho_{3}}^{V_{1}(\varphi)} w(u, v) d \nu d u\right|\left|\frac{\partial^{2} \mathcal{F}}{\partial \psi \partial \varphi}\left(U_{1}(\psi), V_{1}(\varphi)\right)\right| d \varphi d \psi
$$




$$
\begin{aligned}
\leq & \|w\|_{\infty} \int_{0}^{1} \int_{0}^{1}\left|\int_{\rho_{1}}^{U_{1}(\psi)} \int_{\rho_{3}}^{V_{1}(\varphi)} d \nu d u\right|\left|\frac{\partial^{2} \mathcal{F}}{\partial \psi \partial \varphi}\left(U_{1}(\psi), V_{1}(\varphi)\right)\right| d \varphi d \psi \\
\leq & \left(\kappa-\rho_{1}\right)\left(\gamma-\rho_{3}\right)\|w\|_{\infty} \int_{0}^{1} \int_{0}^{1} \psi \varphi\left[\psi \varphi\left|\frac{\partial^{2} \mathcal{F}}{\partial \psi \partial \varphi}(\kappa, \gamma)\right|+\psi(1-\varphi)\left|\frac{\partial^{2} \mathcal{F}}{\partial \psi \partial \varphi}\left(\kappa, \rho_{3}\right)\right|\right. \\
& \left.+(1-\psi) \varphi\left|\frac{\partial^{2} \mathcal{F}}{\partial \psi \partial \varphi}\left(\rho_{1}, \gamma\right)\right|+(1-\psi)(1-\varphi)\left|\frac{\partial^{2} \mathcal{F}}{\partial \psi \partial \varphi}\left(\rho_{1}, \rho_{3}\right)\right|\right] d \varphi d \psi \\
= & \left(\kappa-\rho_{1}\right)\left(\gamma-\rho_{3}\right)\|w\|_{\infty} \\
& \times\left[\frac{1}{9}\left|\frac{\partial^{2} \mathcal{F}}{\partial \psi \partial \varphi}(\kappa, \gamma)\right|+\frac{1}{18}\left|\frac{\partial^{2} \mathcal{F}}{\partial \psi \partial \varphi}\left(\kappa, \rho_{3}\right)\right|+\frac{1}{18}\left|\frac{\partial^{2} \mathcal{F}}{\partial \psi \partial \varphi}\left(\rho_{1}, \gamma\right)\right|\right. \\
& \left.+\frac{1}{36}\left|\frac{\partial^{2} \mathcal{F}}{\partial \psi \partial \varphi}\left(\rho_{1}, \rho_{3}\right)\right|\right] .
\end{aligned}
$$

Similarly, we have

$$
\begin{aligned}
\int_{0}^{1} \int_{0}^{1}\left|\int_{\rho_{1}}^{U_{1}(\psi)} \int_{\rho_{4}}^{V_{2}(\varphi)} w(u, v) d \nu d u\right|\left|\frac{\partial^{2} \mathcal{F}}{\partial \psi \partial \varphi}\left(U_{1}(\psi), V_{2}(\varphi)\right)\right| d \varphi d \psi \\
\leq\left(\kappa-\rho_{1}\right)\left(\rho_{4}-\gamma\right)\|w\|_{\infty} \\
\quad \times\left[\frac{1}{9}\left|\frac{\partial^{2} \mathcal{F}}{\partial \psi \partial \varphi}(\kappa, \gamma)\right|+\frac{1}{18}\left|\frac{\partial^{2} \mathcal{F}}{\partial \psi \partial \varphi}\left(\kappa, \rho_{4}\right)\right|+\frac{1}{18}\left|\frac{\partial^{2} \mathcal{F}}{\partial \psi \partial \varphi}\left(\rho_{1}, \gamma\right)\right|\right. \\
\left.+\frac{1}{36}\left|\frac{\partial^{2} \mathcal{F}}{\partial \psi \partial \varphi}\left(\rho_{1}, \rho_{4}\right)\right|\right], \\
\int_{0}^{1} \int_{0}^{1}\left|\int_{\rho_{2}}^{U_{2}(\psi)} \int_{\rho_{3}}^{V_{1}(\varphi)} w(u, v) d v d u\right|\left|\frac{\partial^{2} \mathcal{F}}{\partial \psi \partial \varphi}\left(U_{2}(\psi), V_{1}(\varphi)\right)\right| d \varphi d \psi \\
\leq \quad\left(\rho_{2}-\kappa\right)\left(\gamma-\rho_{3}\right)\|w\|_{\infty} \\
\quad \times\left[\frac{1}{9}\left|\frac{\partial^{2} \mathcal{F}}{\partial \psi \partial \varphi}(\kappa, \gamma)\right|+\frac{1}{18}\left|\frac{\partial^{2} \mathcal{F}}{\partial \psi \partial \varphi}\left(\kappa, \rho_{3}\right)\right|+\frac{1}{18}\left|\frac{\partial^{2} \mathcal{F}}{\partial \psi \partial \varphi}\left(\rho_{2}, \gamma\right)\right|\right. \\
\left.+\frac{1}{36}\left|\frac{\partial^{2} \mathcal{F}}{\partial \psi \partial \varphi}\left(\rho_{2}, \rho_{3}\right)\right|\right],
\end{aligned}
$$

and

$$
\begin{aligned}
& \int_{0}^{1} \int_{0}^{1}\left|\int_{\rho_{2}}^{U_{2}(\psi)} \int_{\rho_{4}}^{V_{2}(\varphi)} w(u, v) d \nu d u\right|\left|\frac{\partial^{2} \mathcal{F}}{\partial \psi \partial \varphi}\left(U_{2}(\psi), V_{2}(\varphi)\right)\right| d \varphi d \psi \\
& \leq\left(\rho_{2}-\kappa\right)\left(\rho_{4}-\gamma\right)\|w\|_{\infty} \\
& \quad \times\left[\frac{1}{9}\left|\frac{\partial^{2} \mathcal{F}}{\partial \psi \partial \varphi}(\kappa, \gamma)\right|+\frac{1}{18}\left|\frac{\partial^{2} \mathcal{F}}{\partial \psi \partial \varphi}\left(\kappa, \rho_{4}\right)\right|+\frac{1}{18}\left|\frac{\partial^{2} \mathcal{F}}{\partial \psi \partial \varphi}\left(\rho_{2}, \gamma\right)\right|\right. \\
& \left.+\frac{1}{36}\left|\frac{\partial^{2} \mathcal{F}}{\partial \psi \partial \varphi}\left(\rho_{2}, \rho_{4}\right)\right|\right] .
\end{aligned}
$$

If we substitute the inequalities (2.5)-(2.8) in (2.4), then we obtain the required result (2.3). This completes the proof. 
Corollary 1 Under the same assumption of Theorem 3 with $\left|\frac{\partial^{2} \mathcal{F}}{\partial \psi \partial \varphi}(\kappa, \gamma)\right| \leq M,(\kappa, \gamma) \in \Delta$, we have the following weighted Ostrowski type inequality

$$
\begin{aligned}
& \left|\Theta\left(\rho_{1}, \rho_{2}, \rho_{3}, \rho_{4} ; \mathcal{F}, p\right)\right| \\
& \quad \leq \frac{M\left(\rho_{2}-\rho_{1}\right)^{2}\left(\rho_{4}-\rho_{3}\right)^{2}}{m\left(\rho_{1}, \rho_{2} ; \rho_{3}, \rho_{4}\right)}\left[\frac{1}{4}+\frac{\left(\kappa-\frac{\rho_{1}+\rho_{2}}{2}\right)^{2}}{\left(\rho_{2}-\rho_{1}\right)^{2}}\right]\left[\frac{1}{4}+\frac{\left(\gamma-\frac{\rho_{3}+\rho_{4}}{2}\right)^{2}}{\left(\rho_{4}-\rho_{3}\right)^{2}}\right]\|w\|_{\infty} .
\end{aligned}
$$

Remark 1 If we choose $w(\kappa, \gamma)=1$ in Corollary 1 , then Corollary 1 reduces to [14, Theorem 3].

Corollary 2 Under the same assumption of Theorem 3 with $\kappa=\frac{\rho_{1}+\rho_{2}}{2}$ and $\gamma=\frac{\rho_{3}+\rho_{4}}{2}$, we have the following weighted Hermite-Hadamard type inequality

$$
\begin{aligned}
& \mid \mathcal{F}\left(\frac{\rho_{1}+\rho_{2}}{2}, \frac{\rho_{3}+\rho_{4}}{2}\right)+\frac{1}{m\left(\rho_{1}, \rho_{2} ; \rho_{3}, \rho_{4}\right)} \int_{\rho_{1}}^{\rho_{2}} \int_{\rho_{3}}^{\rho_{4}} w(u, v) \mathcal{F}(u, v) d v d u \\
& -\frac{1}{m\left(\rho_{1}, \rho_{2} ; \rho_{3}, \rho_{4}\right)} \int_{\rho_{1}}^{\rho_{2}} \int_{\rho_{3}}^{\rho_{4}} w(u, v) \mathcal{F}\left(u, \frac{\rho_{3}+\rho_{4}}{2}\right) d v d u \\
& -\frac{1}{m\left(\rho_{1}, \rho_{2} ; \rho_{3}, \rho_{4}\right)} \int_{\rho_{1}}^{\rho_{2}} \int_{\rho_{3}}^{\rho_{4}} w(u, v) \mathcal{F}\left(\frac{\rho_{1}+\rho_{2}}{2}, v\right) d v d u \mid \\
& \leq \frac{\left(\rho_{2}-\rho_{1}\right)^{2}\left(\rho_{4}-\rho_{3}\right)^{2}}{576} \frac{\|w\|_{\infty}}{m\left(\rho_{1}, \rho_{2} ; \rho_{3}, \rho_{4}\right)} \\
& \times\left\{16\left|\frac{\partial^{2} \mathcal{F}}{\partial \psi \partial \varphi}\left(\frac{\rho_{1}+\rho_{2}}{2}, \frac{\rho_{3}+\rho_{4}}{2}\right)\right|+4\left|\frac{\partial^{2} \mathcal{F}}{\partial \psi \partial \varphi}\left(\frac{\rho_{1}+\rho_{2}}{2}, \rho_{3}\right)\right|\right. \\
& +4\left|\frac{\partial^{2} \mathcal{F}}{\partial \psi \partial \varphi}\left(\rho_{1}, \frac{\rho_{3}+\rho_{4}}{2}\right)\right|+4\left|\frac{\partial^{2} \mathcal{F}}{\partial \psi \partial \varphi}\left(\frac{\rho_{1}+\rho_{2}}{2}, \rho_{4}\right)\right| \\
& +4\left|\frac{\partial^{2} \mathcal{F}}{\partial \psi \partial \varphi}\left(\rho_{2}, \frac{\rho_{3}+\rho_{4}}{2}\right)\right|+\left|\frac{\partial^{2} \mathcal{F}}{\partial \psi \partial \varphi}\left(\rho_{1}, \rho_{3}\right)\right| \\
& \left.+\left|\frac{\partial^{2} \mathcal{F}}{\partial \psi \partial \varphi}\left(\rho_{1}, \rho_{4}\right)\right|+\left|\frac{\partial^{2} \mathcal{F}}{\partial \psi \partial \varphi}\left(\rho_{2}, \rho_{3}\right)\right|+\left|\frac{\partial^{2} \mathcal{F}}{\partial \psi \partial \varphi}\left(\rho_{2}, \rho_{4}\right)\right|\right\} \\
& \leq \frac{\left(\rho_{2}-\rho_{1}\right)^{2}\left(\rho_{4}-\rho_{3}\right)^{2}}{64} \frac{\|w\|_{\infty}}{m\left(\rho_{1}, \rho_{2} ; \rho_{3}, \rho_{4}\right)} \\
& \times\left[\left|\frac{\partial^{2} \mathcal{F}}{\partial \psi \partial \varphi}\left(\rho_{1}, \rho_{3}\right)\right|+\left|\frac{\partial^{2} \mathcal{F}}{\partial \psi \partial \varphi}\left(\rho_{1}, \rho_{4}\right)\right|+\left|\frac{\partial^{2} \mathcal{F}}{\partial \psi \partial \varphi}\left(\rho_{2}, \rho_{3}\right)\right|+\left|\frac{\partial^{2} \mathcal{F}}{\partial \psi \partial \varphi}\left(\rho_{2}, \rho_{4}\right)\right|\right] .
\end{aligned}
$$

Theorem 4 Let $w$ be as in Theorem 3. If $\left|\frac{\partial^{2} \mathcal{F}}{\partial \psi \partial \varphi}\right|^{q}$ is a co-ordinated convex function on $\Delta$, then for all $(\kappa, \gamma) \in \Delta$, we have the following inequality

$$
\begin{aligned}
& \left|\Theta\left(\rho_{1}, \rho_{2}, \rho_{3}, \rho_{4} ; \mathcal{F}, p\right)\right| \\
& \leq \frac{\|w\|_{\infty}}{m\left(\rho_{1}, \rho_{2} ; \rho_{3}, \rho_{4}\right)(p+1)^{\frac{2}{p}}} \\
& \quad \times\left\{\left(\kappa-\rho_{1}\right)^{2}\left(\gamma-\rho_{3}\right)^{2}\right. \\
& \quad \times\left(\frac { 1 } { 4 } \left[\left|\frac{\partial^{2} \mathcal{F}}{\partial \psi \partial \varphi}(\kappa, \gamma)\right|^{q}+\left|\frac{\partial^{2} \mathcal{F}}{\partial \psi \partial \varphi}\left(\kappa, \rho_{3}\right)\right|^{q}+\left|\frac{\partial^{2} \mathcal{F}}{\partial \psi \partial \varphi}\left(\rho_{1}, \gamma\right)\right|^{q}\right.\right.
\end{aligned}
$$




$$
\begin{aligned}
& \left.\left.+\left|\frac{\partial^{2} \mathcal{F}}{\partial \psi \partial \varphi}\left(\rho_{1}, \rho_{3}\right)\right|^{q}\right]\right)^{\frac{1}{q}} \\
& +\left(\kappa-\rho_{1}\right)^{2}\left(\rho_{4}-\gamma\right)^{2} \\
& \times\left(\frac { 1 } { 4 } \left[\left|\frac{\partial^{2} \mathcal{F}}{\partial \psi \partial \varphi}(\kappa, \gamma)\right|^{q}+\left|\frac{\partial^{2} \mathcal{F}}{\partial \psi \partial \varphi}\left(\kappa, \rho_{4}\right)\right|^{q}+\left|\frac{\partial^{2} \mathcal{F}}{\partial \psi \partial \varphi}\left(\rho_{1}, \gamma\right)\right|^{q}\right.\right. \\
& \left.\left.+\left|\frac{\partial^{2} \mathcal{F}}{\partial \psi \partial \varphi}\left(\rho_{1}, \rho_{4}\right)\right|^{q}\right]\right)^{\frac{1}{q}} \\
& +\left(\rho_{2}-\kappa\right)^{2}\left(\gamma-\rho_{3}\right)^{2} \\
& \times\left(\frac { 1 } { 4 } \left[\left|\frac{\partial^{2} \mathcal{F}}{\partial \psi \partial \varphi}(\kappa, \gamma)\right|^{q}+\left|\frac{\partial^{2} \mathcal{F}}{\partial \psi \partial \varphi}\left(\kappa, \rho_{3}\right)\right|^{q}+\left|\frac{\partial^{2} \mathcal{F}}{\partial \psi \partial \varphi}\left(\rho_{2}, \gamma\right)\right|^{q}\right.\right. \\
& \left.\left.+\left|\frac{\partial^{2} \mathcal{F}}{\partial \psi \partial \varphi}\left(\rho_{2}, \rho_{3}\right)\right|^{q}\right]\right)^{\frac{1}{q}} \\
& +\left(\rho_{2}-\kappa\right)^{2}\left(\rho_{4}-\gamma\right)^{2} \\
& \left.\left.\left.+\left|\frac{\partial^{2} \mathcal{F}}{\partial \psi \partial \varphi}\left(\rho_{2}, \rho_{4}\right)\right|^{q}\right]\right)^{\frac{1}{q}}\right\}, \\
& +\left(\left.\frac{\partial^{2} \mathcal{F}}{\partial \psi \partial \varphi}(\kappa, \gamma)\right|^{q}+\left|\frac{\partial^{2} \mathcal{F}}{\partial \psi \partial \varphi}\left(\kappa, \rho_{4}\right)\right|^{q}+\left|\frac{\partial^{2} \mathcal{F}}{\partial \psi \partial \varphi}\left(\rho_{2}, \gamma\right)\right|^{q}\right.
\end{aligned}
$$

where the mapping $\Theta$ is defined as in (2.1), and $\frac{1}{p}+\frac{1}{q}=1$.

Proof Using the well-known Hölder inequality in (2.4), we obtain

$$
\begin{aligned}
&\left|\Theta\left(\rho_{1}, \rho_{2}, \rho_{3}, \rho_{4} ; \mathcal{F}, p\right)\right| \\
& \leq \frac{\left(\kappa-\rho_{1}\right)\left(\gamma-\rho_{3}\right)}{m\left(\rho_{1}, \rho_{2} ; \rho_{3}, \rho_{4}\right)}\left(\int_{0}^{1} \int_{0}^{1}\left|\int_{\rho_{1}}^{U_{1}(\psi)} \int_{\rho_{3}}^{V_{1}(\varphi)} w(u, v) d v d u\right|^{p} d \varphi d \psi\right)^{\frac{1}{p}} \\
& \times\left(\int_{0}^{1} \int_{0}^{1}\left|\frac{\partial^{2} \mathcal{F}}{\partial \psi \partial \varphi}\left(U_{1}(\psi), V_{1}(\varphi)\right)\right|^{q} d \varphi d \psi\right)^{\frac{1}{q}} \\
&+\frac{\left(\kappa-\rho_{1}\right)\left(\rho_{4}-\gamma\right)}{m\left(\rho_{1}, \rho_{2} ; \rho_{3}, \rho_{4}\right)}\left(\int_{0}^{1} \int_{0}^{1}\left|\int_{\rho_{1}}^{U_{1}(\psi)} \int_{\rho_{4}}^{V_{2}(\varphi)} w(u, v) d v d u\right|^{p} d \varphi d \psi\right)^{\frac{1}{p}} \\
& \times\left(\int_{0}^{1} \int_{0}^{1}\left|\frac{\partial^{2} \mathcal{F}}{\partial \psi \partial \varphi}\left(U_{1}(\psi), V_{2}(\varphi)\right)\right|^{q} d \varphi d \psi\right)^{\frac{1}{q}} \\
&+\frac{\left(\rho_{2}-\kappa\right)\left(\gamma-\rho_{3}\right)}{m\left(\rho_{1}, \rho_{2} ; \rho_{3}, \rho_{4}\right)}\left(\int_{0}^{1} \int_{0}^{1}\left|\int_{\rho_{2}}^{U_{2}(\psi)} \int_{\rho_{3}}^{V_{1}(\varphi)} w(u, v) d \nu d u\right|^{p} d \varphi d \psi\right)^{\frac{1}{p}} \\
& \times\left(\int_{0}^{1} \int_{0}^{1}\left|\frac{\partial^{2} \mathcal{F}}{\partial \psi \partial \varphi}\left(U_{2}(\psi), V_{1}(\varphi)\right)\right|^{q} d \varphi d \psi\right)^{\frac{1}{q}} \\
&+\frac{\left(\rho_{2}-\kappa\right)\left(\rho_{4}-\gamma\right)}{m\left(\rho_{1}, \rho_{2} ; \rho_{3}, \rho_{4}\right)}\left(\int_{0}^{1} \int_{0}^{1}\left|\int_{\rho_{2}}^{U_{2}(\psi)} \int_{\rho_{4}}^{V_{2}(\varphi)} w(u, v) d \nu d u\right|^{p} d \varphi d \psi\right)^{\frac{1}{p}} \\
& \times\left(\int_{0}^{1} \int_{0}^{1}\left|\frac{\partial^{2} \mathcal{F}}{\partial \psi \partial \varphi}\left(U_{2}(\psi), V_{2}(\varphi)\right)\right|^{\frac{1}{q}} d \varphi d \psi\right)^{\frac{1}{q}} \cdot
\end{aligned}
$$


Since $w$ is bounded on $\Delta$, we have

$$
\begin{aligned}
& \int_{0}^{1} \int_{0}^{1}\left|\int_{\rho_{1}}^{U_{1}(\psi)} \int_{\rho_{3}}^{V_{1}(\varphi)} w(u, v) d v d u\right|^{p} d \varphi d \psi \\
& \quad \leq\|w\|_{\infty}^{p} \int_{0}^{1} \int_{0}^{1}\left|\int_{\rho_{1}}^{U_{1}(\psi)} \int_{\rho_{3}}^{V_{1}(\varphi)} d v d u\right|^{p} d \varphi d \psi \\
& =\|w\|_{\infty}^{p}\left(\kappa-\rho_{1}\right)^{p}\left(\gamma-\rho_{3}\right)^{p} \int_{0}^{1} \int_{0}^{1} \varphi^{p} \psi^{p} d \varphi d \psi \\
& =\frac{\left(\kappa-\rho_{1}\right)^{p}\left(\gamma-\rho_{3}\right)^{p}}{(p+1)^{2}}\|w\|_{\infty}^{p} .
\end{aligned}
$$

Similarly, we get

$$
\begin{aligned}
& \int_{0}^{1} \int_{0}^{1}\left|\int_{\rho_{1}}^{U_{1}(\psi)} \int_{\rho_{4}}^{V_{2}(\varphi)} w(u, v) d \nu d u\right|^{p} d \varphi d \psi \leq \frac{\left(\kappa-\rho_{1}\right)^{p}\left(\rho_{4}-\gamma\right)^{p}}{(p+1)^{2}}\|w\|_{\infty}^{p}, \\
& \int_{0}^{1} \int_{0}^{1}\left|\int_{\rho_{2}}^{U_{2}(\psi)} \int_{\rho_{3}}^{V_{1}(\varphi)} w(u, v) d \nu d u\right|^{p} d \varphi d \psi \leq \frac{\left(\rho_{2}-\kappa\right)^{p}\left(\gamma-\rho_{3}\right)^{p}}{(p+1)^{2}}\|w\|_{\infty}^{p},
\end{aligned}
$$

and

$$
\int_{0}^{1} \int_{0}^{1}\left|\int_{\rho_{2}}^{u_{2}(\psi)} \int_{\rho_{4}}^{V_{2}(\varphi)} w(u, v) d \nu d u\right|^{p} d \varphi d \psi \leq \frac{\left(\rho_{2}-\kappa\right)^{p}\left(\rho_{4}-\gamma\right)^{p}}{(p+1)^{2}}\|w\|_{\infty}^{p} .
$$

On the other hand, as $\left|\frac{\partial^{2} \mathcal{F}}{\partial \psi \partial \varphi}\right|^{q}$ is a co-ordinated convex function on $\Delta$, we obtain

$$
\begin{aligned}
& \int_{0}^{1} \int_{0}^{1}\left|\frac{\partial^{2} \mathcal{F}}{\partial \psi \partial \varphi}\left(U_{1}(\psi), V_{1}(\varphi)\right)\right|^{q} d \varphi d \psi \frac{1}{4}\left[\left|\frac{\partial^{2} \mathcal{F}}{\partial \psi \partial \varphi}(\kappa, \gamma)\right|^{q}+\left|\frac{\partial^{2} \mathcal{F}}{\partial \psi \partial \varphi}\left(\kappa, \rho_{3}\right)\right|^{q}+\left|\frac{\partial^{2} \mathcal{F}}{\partial \psi \partial \varphi}\left(\rho_{1}, \gamma\right)\right|^{q}\right. \\
&\left.+\left|\frac{\partial^{2} \mathcal{F}}{\partial \psi \partial \varphi}\left(\rho_{1}, \rho_{3}\right)\right|^{q}\right], \\
& \int_{0}^{1} \int_{0}^{1}\left|\frac{\partial^{2} \mathcal{F}}{\partial \psi \partial \varphi}\left(U_{1}(\psi), V_{2}(\varphi)\right)\right|^{q} d \varphi d \psi \frac{1}{4}\left[\left|\frac{\partial^{2} \mathcal{F}}{\partial \psi \partial \varphi}(\kappa, \gamma)\right|^{q}+\left|\frac{\partial^{2} \mathcal{F}}{\partial \psi \partial \varphi}\left(\kappa, \rho_{4}\right)\right|^{q}+\left|\frac{\partial^{2} \mathcal{F}}{\partial \psi \partial \varphi}\left(\rho_{1}, \gamma\right)\right|^{q}\right. \\
& \quad\left.\left|\frac{\partial^{2} \mathcal{F}}{\partial \psi \partial \varphi}\left(\rho_{1}, \rho_{4}\right)\right|^{q}\right], \\
& \int_{0}^{1} \int_{0}^{1}\left|\frac{\partial^{2} \mathcal{F}}{\partial \psi \partial \varphi}\left(U_{2}(\psi), V_{1}(\varphi)\right)\right|^{q} d \varphi d \psi \\
& \leq \frac{1}{4}\left[\left|\frac{\partial^{2} \mathcal{F}}{\partial \psi \partial \varphi}(\kappa, \gamma)\right|^{q}+\left|\frac{\partial^{2} \mathcal{F}}{\partial \psi \partial \varphi}\left(\kappa, \rho_{3}\right)\right|^{q}+\left|\frac{\partial^{2} \mathcal{F}}{\partial \psi \partial \varphi}\left(\rho_{2}, \gamma\right)\right|^{q}\right. \\
&\left.+\left|\frac{\partial^{2} \mathcal{F}}{\partial \psi \partial \varphi}\left(\rho_{2}, \rho_{3}\right)\right|^{q}\right],
\end{aligned}
$$


and

$$
\begin{aligned}
& \int_{0}^{1} \int_{0}^{1}\left|\frac{\partial^{2} \mathcal{F}}{\partial \psi \partial \varphi}\left(U_{2}(\psi), V_{2}(\varphi)\right)\right|^{q} d \varphi d \psi \\
& \leq \frac{1}{4}\left[\left|\frac{\partial^{2} \mathcal{F}}{\partial \psi \partial \varphi}(\kappa, \gamma)\right|^{q}+\left|\frac{\partial^{2} \mathcal{F}}{\partial \psi \partial \varphi}\left(\kappa, \rho_{4}\right)\right|^{q}+\left|\frac{\partial^{2} \mathcal{F}}{\partial \psi \partial \varphi}\left(\rho_{2}, \gamma\right)\right|^{q}\right. \\
& \left.\quad+\left|\frac{\partial^{2} \mathcal{F}}{\partial \psi \partial \varphi}\left(\rho_{2}, \rho_{4}\right)\right|^{q}\right] .
\end{aligned}
$$

If we substitute the inequalities (2.11)-(2.18) in (2.10), then we obtain the required inequality (2.9).

Corollary 3 Under the same assumption of Theorem 4 with $\left|\frac{\partial^{2} \mathcal{F}}{\partial \psi \partial \varphi}(\kappa, \gamma)\right| \leq M,(\kappa, \gamma) \in \Delta$, then we have the following weighted Ostrowski type inequality

$$
\begin{aligned}
& \left|\Theta\left(\rho_{1}, \rho_{2}, \rho_{3}, \rho_{4} ; \mathcal{F}, p\right)\right| \\
& \quad \leq \frac{4 M\left(\rho_{2}-\rho_{1}\right)^{2}\left(\rho_{4}-\rho_{3}\right)^{2}}{m\left(\rho_{1}, \rho_{2} ; \rho_{3}, \rho_{4}\right)(p+1)^{\frac{2}{p}}}\left[\frac{1}{4}+\frac{\left(\kappa-\frac{\rho_{1}+\rho_{2}}{2}\right)^{2}}{\left(\rho_{2}-\rho_{1}\right)^{2}}\right]\left[\frac{1}{4}+\frac{\left(\gamma-\frac{\rho_{3}+\rho_{4}}{2}\right)^{2}}{\left(\rho_{4}-\rho_{3}\right)^{2}}\right]\|w\|_{\infty} .
\end{aligned}
$$

Remark 2 If we choose $w(\kappa, \gamma)=1$ in Corollary 3 , then Corollary 3 reduces to [14, Theorem 4].

Corollary 4 Under the same assumption of Theorem 4 with $\kappa=\frac{\rho_{1}+\rho_{2}}{2}$ and $\gamma=\frac{\rho_{3}+\rho_{4}}{2}$, then we have the following weighted Hermite-Hadamard type inequality

$$
\begin{aligned}
& \mid \mathcal{F}\left(\frac{\rho_{1}+\rho_{2}}{2}, \frac{\rho_{3}+\rho_{4}}{2}\right)+\frac{1}{m\left(\rho_{1}, \rho_{2} ; \rho_{3}, \rho_{4}\right)} \int_{\rho_{1}}^{\rho_{2}} \int_{\rho_{3}}^{\rho_{4}} w(u, v) \mathcal{F}(u, v) d v d u \\
& -\frac{1}{m\left(\rho_{1}, \rho_{2} ; \rho_{3}, \rho_{4}\right)} \int_{\rho_{1}}^{\rho_{2}} \int_{\rho_{3}}^{\rho_{4}} w(u, v) \mathcal{F}\left(u, \frac{\rho_{3}+\rho_{4}}{2}\right) d v d u \\
& -\frac{1}{m\left(\rho_{1}, \rho_{2} ; \rho_{3}, \rho_{4}\right)} \int_{\rho_{1}}^{\rho_{2}} \int_{\rho_{3}}^{\rho_{4}} w(u, v) \mathcal{F}\left(\frac{\rho_{1}+\rho_{2}}{2}, v\right) d v d u \\
& \leq \frac{\|w\|_{\infty}\left(\rho_{2}-\rho_{1}\right)^{2}\left(\rho_{4}-\rho_{3}\right)^{2}}{2^{4+\frac{2}{q}} \times m\left(\rho_{1}, \rho_{2} ; \rho_{3}, \rho_{4}\right)(p+1)^{\frac{2}{p}}} \\
& \times\left\{\left(\left|\frac{\partial^{2} \mathcal{F}}{\partial \psi \partial \varphi}\left(\frac{\rho_{1}+\rho_{2}}{2}, \frac{\rho_{3}+\rho_{4}}{2}\right)\right|^{q}+\left|\frac{\partial^{2} \mathcal{F}}{\partial \psi \partial \varphi}\left(\frac{\rho_{1}+\rho_{2}}{2}, \rho_{3}\right)\right|^{q}\right.\right. \\
& \left.+\left|\frac{\partial^{2} \mathcal{F}}{\partial \psi \partial \varphi}\left(\rho_{1}, \frac{\rho_{3}+\rho_{4}}{2}\right)\right|^{q}+\left|\frac{\partial^{2} \mathcal{F}}{\partial \psi \partial \varphi}\left(\rho_{1}, \rho_{3}\right)\right|^{q}\right)^{\frac{1}{q}} \\
& +\left(\left|\frac{\partial^{2} \mathcal{F}}{\partial \psi \partial \varphi}\left(\frac{\rho_{1}+\rho_{2}}{2}, \frac{\rho_{3}+\rho_{4}}{2}\right)\right|^{q}+\left|\frac{\partial^{2} \mathcal{F}}{\partial \psi \partial \varphi}\left(\frac{\rho_{1}+\rho_{2}}{2}, \rho_{4}\right)\right|^{q}\right. \\
& \left.+\left|\frac{\partial^{2} \mathcal{F}}{\partial \psi \partial \varphi}\left(\rho_{1}, \frac{\rho_{3}+\rho_{4}}{2}\right)\right|^{q}+\left|\frac{\partial^{2} \mathcal{F}}{\partial \psi \partial \varphi}\left(\rho_{1}, \rho_{4}\right)\right|^{q}\right)^{\frac{1}{q}} \\
& +\left(\left|\frac{\partial^{2} \mathcal{F}}{\partial \psi \partial \varphi}\left(\frac{\rho_{1}+\rho_{2}}{2}, \frac{\rho_{3}+\rho_{4}}{2}\right)\right|^{q}+\left|\frac{\partial^{2} \mathcal{F}}{\partial \psi \partial \varphi}\left(\frac{\rho_{1}+\rho_{2}}{2}, \rho_{3}\right)\right|^{q}\right. \\
& \left.+\left|\frac{\partial^{2} \mathcal{F}}{\partial \psi \partial \varphi}\left(\rho_{2}, \frac{\rho_{3}+\rho_{4}}{2}\right)\right|^{q}+\left|\frac{\partial^{2} \mathcal{F}}{\partial \psi \partial \varphi}\left(\rho_{2}, \rho_{3}\right)\right|^{q}\right)^{\frac{1}{q}}
\end{aligned}
$$




$$
\begin{aligned}
& +\left(\left|\frac{\partial^{2} \mathcal{F}}{\partial \psi \partial \varphi}\left(\frac{\rho_{1}+\rho_{2}}{2}, \frac{\rho_{3}+\rho_{4}}{2}\right)\right|^{q}+\left|\frac{\partial^{2} \mathcal{F}}{\partial \psi \partial \varphi}\left(\frac{\rho_{1}+\rho_{2}}{2}, \rho_{4}\right)\right|^{q}\right. \\
& \left.\left.+\left|\frac{\partial^{2} \mathcal{F}}{\partial \psi \partial \varphi}\left(\rho_{2}, \frac{\rho_{3}+\rho_{4}}{2}\right)\right|^{q}+\left|\frac{\partial^{2} \mathcal{F}}{\partial \psi \partial \varphi}\left(\rho_{2}, \rho_{4}\right)\right|^{q}\right)^{\frac{1}{q}}\right\} .
\end{aligned}
$$

Theorem 5 Let $w$ be as in Theorem 3. If the function $\left|\frac{\partial^{2} \mathcal{F}}{\partial \psi \partial \varphi}\right|^{q}$ is a co-ordinated convex function on $\Delta$, then for all $(\kappa, \gamma) \in \Delta$ and $q \geq 1$, we have the following inequality

$$
\begin{aligned}
\left|\Theta\left(\rho_{1}, \rho_{2}, \rho_{3}, \rho_{4} ; \mathcal{F}, p\right)\right| \\
\leq \frac{\left(\kappa-\rho_{1}\right)^{2}\left(\gamma-\rho_{3}\right)^{2}\|w\|_{\infty}}{4 \times m\left(\rho_{1}, \rho_{2} ; \rho_{3}, \rho_{4}\right)} \\
\quad \times\left(\frac{4}{9}\left|\frac{\partial^{2} \mathcal{F}}{\partial \psi \partial \varphi}(\kappa, \gamma)\right|^{q}+\frac{2}{9}\left|\frac{\partial^{2} \mathcal{F}}{\partial \psi \partial \varphi}\left(\kappa, \rho_{3}\right)\right|^{q}+\frac{2}{9}\left|\frac{\partial^{2} \mathcal{F}}{\partial \psi \partial \varphi}\left(\rho_{1}, \gamma\right)\right|^{q}\right. \\
\left.+\frac{1}{9}\left|\frac{\partial^{2} \mathcal{F}}{\partial \psi \partial \varphi}\left(\rho_{1}, \rho_{3}\right)\right|^{q}\right)^{\frac{1}{q}} \\
+\frac{\left(\kappa-\rho_{1}\right)^{2}\left(\rho_{4}-\gamma\right)^{2}\|w\|_{\infty}}{4 \times m\left(\rho_{1}, \rho_{2} ; \rho_{3}, \rho_{4}\right)} \\
\quad \times\left(\frac{4}{9}\left|\frac{\partial^{2} \mathcal{F}}{\partial \psi \partial \varphi}(\kappa, \gamma)\right|^{q}+\frac{2}{9}\left|\frac{\partial^{2} \mathcal{F}}{\partial \psi \partial \varphi}\left(\kappa, \rho_{4}\right)\right|^{q}+\frac{2}{9}\left|\frac{\partial^{2} \mathcal{F}}{\partial \psi \partial \varphi}\left(\rho_{1}, \gamma\right)\right|^{q}\right. \\
\left.+\frac{1}{9}\left|\frac{\partial^{2} \mathcal{F}}{\partial \psi \partial \varphi}\left(\rho_{1}, \rho_{4}\right)\right|^{q}\right)^{\frac{1}{q}} \\
\left.+\frac{1}{9}\left|\frac{\partial^{2} \mathcal{F}}{\partial \psi \partial \varphi}\left(\rho_{2}, \rho_{4}\right)\right|^{q}\right)^{\frac{1}{q}}, \\
+\frac{\left(\rho_{2}-\kappa\right)^{2}\left(\gamma-\rho_{3}\right)^{2}\|w\|_{\infty}}{4 \times m\left(\rho_{1}, \rho_{2} ; \rho_{3}, \rho_{4}\right)} \\
\left.+\frac{4}{9}\left|\frac{\partial^{2} \mathcal{F}}{\partial \psi \partial \varphi}\left(\rho_{2}, \rho_{3}\right)\right|^{q}\right)^{\frac{1}{q}}\left(\rho_{4}-\gamma\right)^{2}\|w\|_{\infty} \\
+
\end{aligned}
$$

where the mapping $\Theta$ is defined as in (2.1).

Proof By utilizing the power mean inequality in (2.4), we obtain

$$
\begin{aligned}
& \left|\Theta\left(\rho_{1}, \rho_{2}, \rho_{3}, \rho_{4} ; \mathcal{F}, p\right)\right| \\
& \quad \leq \frac{\left(\kappa-\rho_{1}\right)\left(\gamma-\rho_{3}\right)}{m\left(\rho_{1}, \rho_{2} ; \rho_{3}, \rho_{4}\right)}\left(\int_{0}^{1} \int_{0}^{1}\left|\int_{\rho_{1}}^{U_{1}(\psi)} \int_{\rho_{3}}^{V_{1}(\varphi)} w(u, v) d v d u\right| d \varphi d \psi\right)^{1-\frac{1}{q}}
\end{aligned}
$$




$$
\begin{aligned}
& \times\left(\int_{0}^{1} \int_{0}^{1}\left|\int_{\rho_{1}}^{U_{1}(\psi)} \int_{\rho_{3}}^{V_{1}(\varphi)} w(u, v) d v d u\right|\left|\frac{\partial^{2} \mathcal{F}}{\partial \psi \partial \varphi}\left(U_{1}(\psi), V_{1}(\varphi)\right)\right|^{q} d \varphi d \psi\right)^{\frac{1}{q}} \\
& +\frac{\left(\kappa-\rho_{1}\right)\left(\rho_{4}-\gamma\right)}{m\left(\rho_{1}, \rho_{2} ; \rho_{3}, \rho_{4}\right)}\left(\int_{0}^{1} \int_{0}^{1}\left|\int_{\rho_{1}}^{U_{1}(\psi)} \int_{\rho_{4}}^{V_{2}(\varphi)} w(u, v) d v d u\right| d \varphi d \psi\right)^{1-\frac{1}{q}} \\
& \times\left(\int_{0}^{1} \int_{0}^{1}\left|\int_{\rho_{1}}^{U_{1}(\psi)} \int_{\rho_{4}}^{V_{2}(\varphi)} w(u, v) d v d u\right|\left|\frac{\partial^{2} \mathcal{F}}{\partial \psi \partial \varphi}\left(U_{1}(\psi), V_{2}(\varphi)\right)\right|^{q} d \varphi d \psi\right)^{\frac{1}{q}} \\
& +\frac{\left(\rho_{2}-\kappa\right)\left(\gamma-\rho_{3}\right)}{m\left(\rho_{1}, \rho_{2} ; \rho_{3}, \rho_{4}\right)}\left(\int_{0}^{1} \int_{0}^{1}\left|\int_{\rho_{2}}^{U_{2}(\psi)} \int_{\rho_{3}}^{V_{1}(\varphi)} w(u, v) d v d u\right| d \varphi d \psi\right)^{1-\frac{1}{q}} \\
& \times\left(\int_{0}^{1} \int_{0}^{1}\left|\int_{\rho_{2}}^{U_{2}(\psi)} \int_{\rho_{3}}^{V_{1}(\varphi)} w(u, v) d v d u\right|\left|\frac{\partial^{2} \mathcal{F}}{\partial \psi \partial \varphi}\left(U_{2}(\psi), V_{1}(\varphi)\right)\right|^{q} d \varphi d \psi\right)^{\frac{1}{q}} \\
& +\frac{\left(\rho_{2}-\kappa\right)\left(\rho_{4}-\gamma\right)}{m\left(\rho_{1}, \rho_{2} ; \rho_{3}, \rho_{4}\right)}\left(\int_{0}^{1} \int_{0}^{1}\left|\int_{\rho_{2}}^{U_{2}(\psi)} \int_{\rho_{4}}^{V_{2}(\varphi)} w(u, v) d v d u\right| d \varphi d \psi\right)^{1-\frac{1}{q}} \\
& \times\left(\int_{0}^{1} \int_{0}^{1}\left|\int_{\rho_{2}}^{U_{2}(\psi)} \int_{\rho_{4}}^{V_{2}(\varphi)} w(u, v) d v d u\right|\left|\frac{\partial^{2} \mathcal{F}}{\partial \psi \partial \varphi}\left(U_{2}(\psi), V_{2}(\varphi)\right)\right|^{q} d \varphi d \psi\right)^{\frac{1}{q}} \\
& \leq \frac{\left(\kappa-\rho_{1}\right)^{2}\left(\gamma-\rho_{3}\right)^{2}\|w\|_{\infty}}{4^{1-\frac{1}{q}} \times m\left(\rho_{1}, \rho_{2} ; \rho_{3}, \rho_{4}\right)}\left(\int_{0}^{1} \int_{0}^{1} \psi \varphi\left|\frac{\partial^{2} \mathcal{F}}{\partial \psi \partial \varphi}\left(U_{1}(\psi), V_{1}(\varphi)\right)\right|^{q} d \varphi d \psi\right)^{\frac{1}{q}} \\
& +\frac{\left(\kappa-\rho_{1}\right)^{2}\left(\rho_{4}-\gamma\right)^{2}\|w\|_{\infty}}{4^{1-\frac{1}{q}} \times m\left(\rho_{1}, \rho_{2} ; \rho_{3}, \rho_{4}\right)}\left(\int_{0}^{1} \int_{0}^{1} \psi \varphi\left|\frac{\partial^{2} \mathcal{F}}{\partial \psi \partial \varphi}\left(U_{1}(\psi), V_{2}(\varphi)\right)\right|^{q} d \varphi d \psi\right)^{\frac{1}{q}} \\
& +\frac{\left(\rho_{2}-\kappa\right)^{2}\left(\gamma-\rho_{3}\right)^{2}\|w\|_{\infty}}{4^{1-\frac{1}{q}} \times m\left(\rho_{1}, \rho_{2} ; \rho_{3}, \rho_{4}\right)}\left(\int_{0}^{1} \int_{0}^{1} \psi \varphi\left|\frac{\partial^{2} \mathcal{F}}{\partial \psi \partial \varphi}\left(U_{2}(\psi), V_{1}(\varphi)\right)\right|^{q} d \varphi d \psi\right)^{\frac{1}{q}} \\
& +\frac{\left(\rho_{2}-\kappa\right)^{2}\left(\rho_{4}-\gamma\right)^{2}\|w\|_{\infty}}{4^{1-\frac{1}{q}} \times m\left(\rho_{1}, \rho_{2} ; \rho_{3}, \rho_{4}\right)}\left(\int_{0}^{1} \int_{0}^{1} \psi \varphi\left|\frac{\partial^{2} \mathcal{F}}{\partial \psi \partial \varphi}\left(U_{2}(\psi), V_{2}(\varphi)\right)\right|^{q} d \varphi d \psi\right)^{\frac{1}{q}} .
\end{aligned}
$$

Since $\left|\frac{\partial^{2} \mathcal{F}}{\partial \psi \partial \varphi}\right|^{q}$ is a co-ordinated convex function on $\Delta$, we have the following inequalities

$$
\begin{aligned}
& \left(\int_{0}^{1} \int_{0}^{1} \psi \varphi\left|\frac{\partial^{2} \mathcal{F}}{\partial \psi \partial \varphi}\left(U_{1}(\psi), V_{1}(\varphi)\right)\right|^{q} d \varphi d \psi\right)^{\frac{1}{q}} \\
& \leq\left(\frac{1}{9}\left|\frac{\partial^{2} \mathcal{F}}{\partial \psi \partial \varphi}(\kappa, \gamma)\right|^{q}+\frac{1}{18}\left|\frac{\partial^{2} \mathcal{F}}{\partial \psi \partial \varphi}\left(\kappa, \rho_{3}\right)\right|^{q}+\frac{1}{18}\left|\frac{\partial^{2} \mathcal{F}}{\partial \psi \partial \varphi}\left(\rho_{1}, \gamma\right)\right|^{q}\right. \\
& \left.\quad+\frac{1}{36}\left|\frac{\partial^{2} \mathcal{F}}{\partial \psi \partial \varphi}\left(\rho_{1}, \rho_{3}\right)\right|^{q}\right)^{\frac{1}{q}}, \\
& \left(\int_{0}^{1} \int_{0}^{1} \psi \varphi\left|\frac{\partial^{2} \mathcal{F}}{\partial \psi \partial \varphi}\left(U_{1}(\psi), V_{2}(\varphi)\right)\right|^{q} d \varphi d \psi\right)^{\frac{1}{q}} \\
& \leq\left(\frac{1}{9}\left|\frac{\partial^{2} \mathcal{F}}{\partial \psi \partial \varphi}(\kappa, \gamma)\right|^{q}+\frac{1}{18}\left|\frac{\partial^{2} \mathcal{F}}{\partial \psi \partial \varphi}\left(\kappa, \rho_{4}\right)\right|^{q}+\frac{1}{18}\left|\frac{\partial^{2} \mathcal{F}}{\partial \psi \partial \varphi}\left(\rho_{1}, \gamma\right)\right|^{q}\right. \\
& \left.\quad+\frac{1}{36}\left|\frac{\partial^{2} \mathcal{F}}{\partial \psi \partial \varphi}\left(\rho_{1}, \rho_{4}\right)\right|^{q}\right)^{\frac{1}{q}}, \\
& \left(\int_{0}^{1} \int_{0}^{1} \psi \varphi\left|\frac{\partial^{2} \mathcal{F}}{\partial \psi \partial \varphi}\left(U_{2}(\psi), V_{1}(\varphi)\right)\right|^{q} d \varphi d \psi\right)^{\frac{1}{q}}
\end{aligned}
$$




$$
\begin{aligned}
\leq & \left(\frac{1}{9}\left|\frac{\partial^{2} \mathcal{F}}{\partial \psi \partial \varphi}(\kappa, \gamma)\right|^{q}+\frac{1}{18}\left|\frac{\partial^{2} \mathcal{F}}{\partial \psi \partial \varphi}\left(\kappa, \rho_{3}\right)\right|^{q}+\frac{1}{18}\left|\frac{\partial^{2} \mathcal{F}}{\partial \psi \partial \varphi}\left(\rho_{2}, \gamma\right)\right|^{q}\right. \\
& \left.+\frac{1}{36}\left|\frac{\partial^{2} \mathcal{F}}{\partial \psi \partial \varphi}\left(\rho_{2}, \rho_{3}\right)\right|^{q}\right)^{\frac{1}{q}}
\end{aligned}
$$

and

$$
\begin{aligned}
& \left(\int_{0}^{1} \int_{0}^{1} \psi \varphi\left|\frac{\partial^{2} \mathcal{F}}{\partial \psi \partial \varphi}\left(U_{2}(\psi), V_{2}(\varphi)\right)\right|^{q} d \varphi d \psi\right)^{\frac{1}{q}} \\
& \leq\left(\frac{1}{9}\left|\frac{\partial^{2} \mathcal{F}}{\partial \psi \partial \varphi}(\kappa, \gamma)\right|^{q}+\frac{1}{18}\left|\frac{\partial^{2} \mathcal{F}}{\partial \psi \partial \varphi}\left(\kappa, \rho_{4}\right)\right|^{q}+\frac{1}{18}\left|\frac{\partial^{2} \mathcal{F}}{\partial \psi \partial \varphi}\left(\rho_{2}, \gamma\right)\right|^{q}\right. \\
& \left.\quad+\frac{1}{36}\left|\frac{\partial^{2} \mathcal{F}}{\partial \psi \partial \varphi}\left(\rho_{2}, \rho_{4}\right)\right|^{q}\right)^{\frac{1}{q}} .
\end{aligned}
$$

By utilizing the equalities (2.21)-(2.24) in (2.20), we obtain the desired inequality (2.19). This completes the proof.

Remark 3 Under the same assumption of Theorem 5 with $\left|\frac{\partial^{2} \mathcal{F}}{\partial \psi \partial \varphi}(\kappa, \gamma)\right| \leq M,(\kappa, \gamma) \in \Delta$, then Theorem 5 reduces to Corollary 1.

Corollary 5 Under the same assumption of Theorem 5 with $\kappa=\frac{\rho_{1}+\rho_{2}}{2}$ and $\gamma=\frac{\rho_{3}+\rho_{4}}{2}$, we have the following weighted Hermite-Hadamard type inequality

$$
\begin{aligned}
\mid \mathcal{F}( & \left.\frac{\rho_{1}+\rho_{2}}{2}, \frac{\rho_{3}+\rho_{4}}{2}\right)+\frac{1}{m\left(\rho_{1}, \rho_{2} ; \rho_{3}, \rho_{4}\right)} \int_{\rho_{1}}^{\rho_{2}} \int_{\rho_{3}}^{\rho_{4}} w(u, v) \mathcal{F}(u, v) d v d u \\
& -\frac{1}{m\left(\rho_{1}, \rho_{2} ; \rho_{3}, \rho_{4}\right)} \int_{\rho_{1}}^{\rho_{2}} \int_{\rho_{3}}^{\rho_{4}} w(u, v) \mathcal{F}\left(u, \frac{\rho_{3}+\rho_{4}}{2}\right) d v d u \\
& -\frac{1}{m\left(\rho_{1}, \rho_{2} ; \rho_{3}, \rho_{4}\right)} \int_{\rho_{1}}^{\rho_{2}} \int_{\rho_{3}}^{\rho_{4}} w(u, v) \mathcal{F}\left(\frac{\rho_{1}+\rho_{2}}{2}, v\right) d v d u \mid \\
\leq & \frac{\left(\rho_{2}-\rho_{1}\right)^{2}\left(\rho_{4}-\rho_{3}\right)^{2}\|w\|_{\infty}}{64 \times m\left(\rho_{1}, \rho_{2} ; \rho_{3}, \rho_{4}\right)} \\
& \times\left[\left(\frac{4}{9}\left|\frac{\partial^{2} \mathcal{F}}{\partial \psi \partial \varphi}\left(\frac{\rho_{1}+\rho_{2}}{2}, \frac{\rho_{3}+\rho_{4}}{2}\right)\right|^{q}+\frac{2}{9}\left|\frac{\partial^{2} \mathcal{F}}{\partial \psi \partial \varphi}\left(\frac{\rho_{1}+\rho_{2}}{2}, \rho_{3}\right)\right|^{q}\right.\right. \\
& \left.+\frac{2}{9}\left|\frac{\partial^{2} \mathcal{F}}{\partial \psi \partial \varphi}\left(\rho_{1}, \frac{\rho_{3}+\rho_{4}}{2}\right)\right|^{q}+\frac{1}{9}\left|\frac{\partial^{2} \mathcal{F}}{\partial \psi \partial \varphi}\left(\rho_{1}, \rho_{3}\right)\right|^{q}\right)^{\frac{1}{q}} \\
& +\left(\frac{4}{9}\left|\frac{\partial^{2} \mathcal{F}}{\partial \psi \partial \varphi}\left(\frac{\rho_{1}+\rho_{2}}{2}, \frac{\rho_{3}+\rho_{4}}{2}\right)\right|^{q}+\frac{2}{9}\left|\frac{\partial^{2} \mathcal{F}}{\partial \psi \partial \varphi}\left(\frac{\rho_{1}+\rho_{2}}{2}, \rho_{4}\right)\right|^{q}\right. \\
& \left.+\frac{2}{9}\left|\frac{\partial^{2} \mathcal{F}}{\partial \psi \partial \varphi}\left(\rho_{1}, \frac{\rho_{3}+\rho_{4}}{2}\right)\right|^{q}+\frac{1}{9}\left|\frac{\partial^{2} \mathcal{F}}{\partial \psi \partial \varphi}\left(\rho_{1}, \rho_{4}\right)\right|^{q}\right)^{\frac{1}{q}} \\
& +\left(\frac{4}{9}\left|\frac{\partial^{2} \mathcal{F}}{\partial \psi \partial \varphi}\left(\frac{\rho_{1}+\rho_{2}}{2}, \frac{\rho_{3}+\rho_{4}}{2}\right)\right|^{q}+\frac{2}{9}\left|\frac{\partial^{2} \mathcal{F}}{\partial \psi \partial \varphi}\left(\frac{\rho_{1}+\rho_{2}}{2}, \rho_{3}\right)\right|^{q}\right. \\
& \left.+\left(\frac{2}{9}\left|\frac{\partial^{2} \mathcal{F}}{\partial \psi \partial \varphi}\left(\rho_{2}, \frac{\rho_{3}+\rho_{4}}{2}\right)\right|^{q}+\frac{1}{9}\left|\frac{\partial^{2} \mathcal{F}}{\partial \psi \partial \varphi}\left(\rho_{2}, \rho_{3}\right)\right|^{q}\right)\right)^{\frac{1}{q}}
\end{aligned}
$$




$$
\begin{aligned}
& +\left(\frac{4}{9}\left|\frac{\partial^{2} \mathcal{F}}{\partial \psi \partial \varphi}\left(\frac{\rho_{1}+\rho_{2}}{2}, \frac{\rho_{3}+\rho_{4}}{2}\right)\right|^{q}+\frac{2}{9}\left|\frac{\partial^{2} \mathcal{F}}{\partial \psi \partial \varphi}\left(\frac{\rho_{1}+\rho_{2}}{2}, \rho_{4}\right)\right|^{q}\right. \\
& \left.\left.+\frac{2}{9}\left|\frac{\partial^{2} \mathcal{F}}{\partial \psi \partial \varphi}\left(\rho_{2}, \frac{\rho_{3}+\rho_{4}}{2}\right)\right|^{q}+\frac{1}{9}\left|\frac{\partial^{2} \mathcal{F}}{\partial \psi \partial \varphi}\left(\rho_{2}, \rho_{4}\right)\right|^{q}\right)^{\frac{1}{q}}\right]
\end{aligned}
$$

\section{Conclusion}

In this paper, we consider the identity given by Ylldiz and Sarıkaya in [30] to obtain some weighted Ostrowski type inequalities for co-ordinated convex functions. We also present some weighted Hermite-Hadamard type inequalities by the special cases of our main results. In future works, the authors can try to generalize their results by utilizing different kinds of co-ordinated convex function classes.

\section{Acknowledgements}

The authors would like to express their sincere thanks to the editor and the anonymous reviewers for their helpful comments and suggestions.

\section{Funding}

There is no funding for this work.

Availability of data and materials

Not applicable.

\section{Declarations}

\section{Competing interests}

The author declares that they have no competing interests.

\section{Authors' contributions}

All authors contributed equally to the writing of this paper. All authors read and approved the final manuscript.

\section{Publisher's Note}

Springer Nature remains neutral with regard to jurisdictional claims in published maps and institutional affiliations.

Received: 18 May 2021 Accepted: 22 December 2021 Published online: 05 January 2022

\section{References}

1. Akkurt, A., Sarikaya, M.Z., Budak, H., Yildirim, H.: On the Hadamard's type inequalities for co-ordinated convex functions via fractional integrals. J. King Saud Univ., Sci. 29, 380-387 (2017)

2. Alomari, M., Darus, M.: The Hadamards inequality for $\varphi$-convex function of 2 -variables on the coordinates. Int. J. Math Anal. 2(13), 629-638 (2008)

3. Alomari, M., Darus, M.: Fejér inequality for double integrals. Facta Univ., Ser. Math. Inform. 24, 15-28 (2009)

4. Bakula, M.K.: An improvement of the Hermite-Hadamard inequality for functions convex on the coordinates. Aust. J. Math. Anal. Appl. 11(1), 1-7 (2014)

5. Barnett, N.S., Dragomir, S.S.: An Ostrowski type inequality for double integrals and applications for cubature formulae. Soochow J. Math. 27(1), 109-114 (2001)

6. Dragomir, S.S.: On Hadamards inequality for convex functions on the co-ordinates in a rectangle from the plane. Taiwan. J. Math. 4, 775-788 (2001)

7. Dragomir, S.S., Pearce, C.E.M.: Selected Topics On Hermite-Hadamard Inequalities and Applications. RGMIA Monographs, Victoria University (2000)

8. Erden, S., Sarıkaya, M.Z.: On the Hermite-Hadamard's and Ostrowski's inequalities for the co-ordinated convex functions. New Trends Math. Sci. 5(3), 33-45 (2017)

9. Farid, G., Marwan, M., Ur Rehman, A.: Fejer-Hadamard inequlality for convex functions on the co-ordinates in a rectangle from the plane. Int. J. Anal. Appl. 10(1), 40-47 (2016)

10. Latif, M.A., Dragomir, S.S.: On some new inequalities for differentiable co-ordinated convex functions. J. Inequal. Appl. 2012, 28 (2012)

11. Latif, M.A., Dragomir, S.S., Momoniat, E.: Weighted generalization of some integral inequalities for differentiable co-ordinated convex functions. Sci. Bull. "Politeh." Univ. Buchar., Ser. A, Appl. Math. Phys. 78(4), 197-210 (2016)

12. Latif, M.A., Dragomir, S.S., Momoniat, E.: Generalization of some inequalities for differentiable co-ordinated convex functions with applications. Moroccan J. Pure Appl. Anal. 2(1), 12-32 (2016)

13. Latif, M.A., Hussain, S., Dragomir, S.S.: On some new Fejer-type inequalities for coordinated convex functions. TJMM 3(2), 57-80 (2011)

14. Latif, M.A., Hussain, S., Dragomir, S.S.: New Ostrowki type inequalities for co-ordinated convex functions. TJMM 4(2), 125-136 (2012) 
15. Ostrowski, A.M.: Über die absolutabweichung einer differentiebaren funktion von ihrem integralmitelwert. Comment. Math. Helv. 10, 226-227 (1938)

16. Ozdemir, M.E., Kavurmacı, H., Akdemir, A.O.: Inequalities for convex and $\varphi$-convex functions on $\Delta:=[a, b] \times[c, d]$. J. Inequal. Appl. 2012, 20 (2012)

17. Ozdemir, M.E., Yildiz, C., Akdemir, A.O.: On the co-ordinated convex functions. Appl. Math. Inf. Sci. 8(3), 1085-1091 (2014)

18. Pečarić, J.E., Proschan, F., Tong, Y.L.: Convex Functions, Partial Orderings and Statistical Applications. Academic Press, Boston (1992)

19. Sarikaya, M.Z:: Some inequalities for differentiable co-ordinated convex mappings. Asian-Eur. J. Math. 8(3), 1550058 (2015)

20. Sarikaya, M.Z:: On the generalized weighted integral inequality for double integrals. Ann. Alexandru loan Cuza Univ.-Math. 61(1), 169-179 (2015)

21. Sarikaya, M.Z., Budak, H., Yaldiz, H.: Cebysev type inequalities for co-ordinated convex functions. Pure Appl. Math. Lett. 2, 36-40 (2014)

22. Sarikaya, M.Z., Budak, H., Yaldiz, H.: Some new Ostrowski type inequalities for co-ordinated convex functions. Turk. J. Anal. Number Theory 2(5), 176-182 (2014)

23. Sarikaya, M.Z., Ogulmus, H., Demircan, T:: On the generalized weighted integral inequality for double integrals. Int. J. Mod. Math. Sci. 10(1), 90-102 (2014)

24. Sarikaya, M.Z., Ogunmez, H.: On the weighted Ostrowski type integral inequality for double integrals. Arab. J. Sci. Eng. 36, 1153-1160 (2011)

25. Sarikaya, M.Z., Set, E., Ozdemir, M.E., Dragomir, S.S.: New some Hadamard's type inequalities for co-ordinated convex functions. Tamsui Oxf. J. Inf. Math. Sci. 28(2), 137-152 (2012)

26. Set, E., Özdemir, M.E., Dragomir, S.S.: On the Hermite-Hadamard inequality and other integral inequalities involving two functions. J. Inequal. Appl. 9, Article ID 148102 (2010)

27. Wang, D.Y., Tseng, K.L., Yang, G.S.: Some Hadamard's inequalities for co-ordinated convex functions in a rectangle from the plane. Taiwan. J. Math. 11, 63-73 (2007)

28. Xiang, R., Chen, F.: On some integral inequalities related to Hermite-Hadamard-Fejér inequalities for coordinated convex functions. Chin. J. Math. 2014, Article ID 796132 (2014)

29. Xue, Q., Zhu, J., Liu, W.: A new generalization of Ostrowski-type inequality involving functions of two independent variables. Comput. Math. Appl. 60, 2219-2224 (2010)

30. Yildiz, M.K., Sarikaya, M.Z.: On the generalized Ostrowski type integral inequality for double integrals. Int. J. Anal. Appl. 13(1), 64-69 (2017)

\section{Submit your manuscript to a SpringerOpen ${ }^{\circ}$ journal and benefit from:}

- Convenient online submission

- Rigorous peer review

- Open access: articles freely available online

- High visibility within the field

- Retaining the copyright to your article

Submit your next manuscript at $\boldsymbol{\nabla}$ springeropen.com 